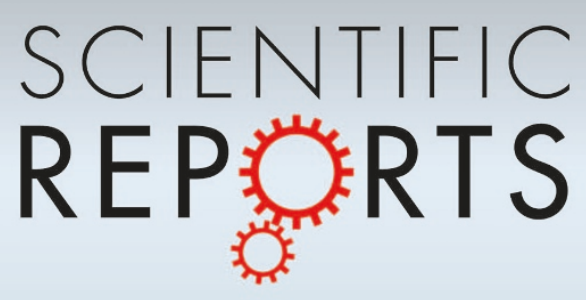

OPEN

SUBJECT AREAS:

COMPUTER SCIENCE

INFORMATION TECHNOLOGY

COMPLEX NETWORKS

APPLIED MATHEMATICS

Received

12 October 2014

Accepted

24 November 2014

Published

12 December 2014

Correspondence and requests for materials should be addressed to

Z.Z.Z. (zhangzz@ fudan.edu.cn)

\title{
Effects of reciprocity on random walks in weighted networks
}

\author{
Zhongzhi Zhang ${ }^{1,2}$, Huan $\mathrm{Li}^{1,2}$ \& Yibin Sheng ${ }^{1,2}$
}

\begin{abstract}
${ }^{1}$ School of Computer Science, Fudan University, Shanghai 200433, China, ${ }^{2}$ Shanghai Key Lab of Intelligent Information Processing, Fudan University, Shanghai 200433, China.
\end{abstract}

\begin{abstract}
It has been recently reported that the reciprocity of real-life weighted networks is very pronounced, however its impact on dynamical processes is poorly understood. In this paper, we study random walks in a scale-free directed weighted network with a trap at the central hub node, where the weight of each directed edge is dominated by a parameter controlling the extent of network reciprocity. We derive two expressions for the mean first passage time (MFPT) to the trap, by using two different techniques, the results of which agree well with each other. We also analytically determine all the eigenvalues as well as their multiplicities for the fundamental matrix of the dynamical process, and show that the largest eigenvalue has an identical dominant scaling as that of the MFPT.We find that the weight parameter has a substantial effect on the MFPT, which behaves as a power-law function of the system size with the power exponent dependent on the parameter, signaling the crucial role of reciprocity in random walks occurring in weighted networks.
\end{abstract}

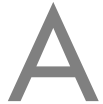

$s$ an emerging science, complex networks have witnessed substantial progress in the past years ${ }^{1}$. One of the ultimate goals in the study of complex networks is to uncover the influences of various structural properties on the function or dynamical processes taking place on them. Among different dynamical processes, random walks lie at the core, since they are a fundamental mechanism for a wealth of other dynamic processes, such as navigation ${ }^{2}$, search ${ }^{3,4}$, and cooperative control ${ }^{5}$. Except for the importance in the area of network science, random walks also provide a paradigmatic model for analyzing and understanding a large variety of real-world phenomena, for example, animal ${ }^{6}$ and human ${ }^{7}$ mobility. Thus far, random walks have found numerous applications $s^{8}$ in many aspects of interdisciplinary sciences, including image segmentation ${ }^{9}$, community detection ${ }^{10,11}$, collaborative recommendation ${ }^{12}$, and signal propagation in proteins ${ }^{13}$ to name a few.

A highly desirable quantity for random walks is first passage time (FPT) ${ }^{14}$, defined as the expected time for a random walker going from a starting node to a given target averaged over all possible trajectories. The mean of FPTs over all starting nodes to the target is called mean first passage time (MFPT), which is an important characteristic of random walks due to the first encounter properties in numerous realistic situations. In the past years, the study of MFPT has triggered an increasing attention from the scientific community ${ }^{15,16}$. One focus of theoretical activity is to develop general methods to efficiently compute $\mathrm{MFPT}^{17-20}$. Another direction is to unveil how the behavior of MFPT is affected by different structural properties of the underlying systems, such as heterogeneity of degree ${ }^{21}$ or strength ${ }^{22}$, fractality ${ }^{23}$, and modularity ${ }^{24}$.

Previous studies proposed several frameworks for evaluating MFPT and uncovered the discernible effects of some nontrivial structural aspects on the target search efficiency measured by MFPT. However, most existing works ignore the impact of link reciprocity, the tendency of node pairs to form mutual connection in directed networks, on the behavior of random walks, despite the fact that reciprocity is a common characteristic of many realistic networks ${ }^{25}$, such as the World-Wide $\mathrm{Web}^{26}$, e-mail networks ${ }^{27,28}$, and world trade web ${ }^{29}$. In addition to binary networks, the nontrivial pattern reciprocity is also ubiquitous in real-life systems described by weighted networks ${ }^{30-32}$. It has been shown the ubiquitous link reciprocity strongly affects dynamical processes in binary networks, for example, spread of computer viruses ${ }^{28}$ or information ${ }^{33}$, and percolation ${ }^{34}$. By contrast, the influence of reciprocity on dynamical processes in weighted networks has attracted much less attention, although it is suggested that reciprocity could play a crucial role in network dynamics. In particular, the lack of analytical results in this field limits our understanding of the impact of weight reciprocity on the function of weighted networks ${ }^{32}$.

In this paper, we propose a weighted directed scale-free network through replacing each edge in the previous binary network ${ }^{35,36}$ by double links with opposite directions and different weights. In the weighted network, the link weights are adjusted by a parameter characterizing the weight reciprocity of network. We then study random walks in the weighted network in the presence of a perfect trap at the central large-degree node. During the process of random walks, the transition probability is dependent on the weight parameter. We derive two 
formulas for the MFPT to the target by using two disparate approaches, the results of which completely agree with each other. We also determine all the eigenvalues and their multiplicities of the fundamental matrix characterizing the random-walk process, and show that the largest eigenvalue has the same leading scaling as that of the MFPT. The obtained results demonstrate that the behavior of MFPT to the trap depends on the weighted parameter, implying a drastic influence of the weight reciprocity on random walks defining on weighted networks.

\section{Results}

Network models and properties. Before introducing the weighted directed network with scale-free fractal properties. We first give a brief introduction to a binary scale-free fractal network, which has the same topology as the weighted network.

Model and properties of binary network. The binary network is constructed in an iterative way ${ }^{35,36}$. Let $F_{g}(g \geq 0)$ represent the network after $g$ iterations (generations). For $g=0, F_{0}$ is an edge linked by two nodes. In each successive iteration $g \geq 1, F_{g}$ is constructed from $F_{g-1}$ by performing the following operations on every existing edge in $F_{g-1}$ as shown in Fig. 1: two new nodes (called external nodes) are firstly created and attached, respectively, to both endpoints of the edge; then, the edge is broken, another new node (referred to as an internal node) is placed in its middle and linked to both endpoints of the original edge. Figure 2 illustrates the first several construction processes of the network. The structure of $F_{g}$ is enciphered in its adjacency matrix $A_{g}$, the entries $A_{g}(i, j)$ of which are defined by $A_{g}(i, j)=1$ if two nodes $i$ and $j$ are adjacent in $F_{g}$, or $A_{g}(i, j)=0$ otherwise.

The particular construction of the network allows to calculate exactly its relevant properties. At each generation $g_{i}\left(g_{i} \geq 1\right)$, the number of newly created nodes is $\Upsilon_{g_{i}}=3 \cdot 4^{g_{i}-1}$. Let $\bar{\Lambda}_{g_{i}}$ be the set of nodes generated at iteration $g_{i}$, then $\bar{\Lambda}_{g_{i}}$ can be further classified into two sets $\bar{\Lambda}_{g_{i, \text { ext }}}$ and $\bar{\Lambda}_{g_{i, \text { int }}}$ satisfying $\bar{\Lambda}_{g_{i}}=\bar{\Lambda}_{g_{i} \text {,ext }} \cup \bar{\Lambda}_{g_{i} \text {,int }}$, among which $\bar{\Lambda}_{g_{i} \text {,ext }}$ is the set of external nodes and $\bar{\Lambda}_{g_{i}, \text { int }}$ is the set of internal nodes. We use $|\Omega|$ to stand for the cardinality of a set $\Omega$. Because $\left|\bar{\Lambda}_{g_{i} \text { ext }}\right|=2\left|\bar{\Lambda}_{g_{i}, \text { in }}\right|$, it is easy to derive $\left|\bar{\Lambda}_{g_{i}, \text { int }}\right|=4^{g_{i}-1}$ and $\left|\bar{\Lambda}_{g_{i} \text {,ext }}\right|=$ $2 \cdot 4^{g_{i}-1}$. We represent the set of nodes in $F_{g}$ as $\Lambda_{g}$. Hence, the number of nodes and edges in $F_{g}$ is $N_{g}=\left|\Lambda_{g}\right|=\sum_{g_{i}=0}^{g} \Upsilon_{g_{i}}=4^{g}+1$ and $E_{g}=$ $N_{g}-1=4^{g}$, respectively. Let $k_{i}(g)$ denote the degree of an arbitrary node $i$ in $F_{g}$ that was generated at generation $g_{i}\left(g_{i} \geq 0\right)$, then $k_{i}(g+1)$ $=2 k_{i}(g)$. Hence, after each new iteration the degree of every node doubles.

This resultant network displays the remarkable scale-free ${ }^{37}$ and fractal $^{38}$ features as observed in diverse real-life systems. It has a power law degree distribution with an exponent 3 , and its fractal dimension is 2 .

Model and properties of weighted directed network. The above introduced binary network $F_{g}$ can be extended to a weighted directed network with nonnegative and asymmetrical edge weights. Let $\vec{F}_{g}$ denote the weighted directed network corresponding to $F_{g}$. Both $\vec{F}_{g}$ and $F_{g}$ have an identical topological structure. The only difference between $\vec{F}_{g}$ and $F_{g}$ is that every undirected edge in $F_{g}$ is replaced by two directed edges with opposite directions and distinct positive weights. We use $W_{g}$ to represent the nonnegative and asymmetrical

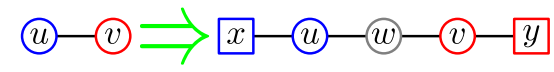

Figure 1 | Illustration of construction of the binary network. The next generation is obtained from current generation by replacing each edge with the cluster on the right-hand side of the arrow, where $w$ is a new internal node, while $x$ and $y$ are external nodes.

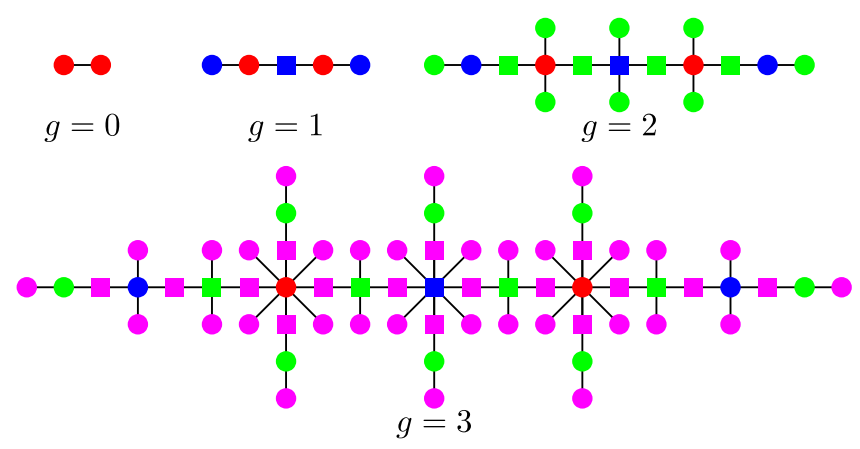

Figure $2 \mid$ Iterative growth processes for the first several generations.

weight matrix for $\vec{F}_{g}$ such that $W_{i j}(g)>0$ if and only if there is a directed edge (arc) pointing to node $j$ from node $i$. The weight of each arc in the weighted directed network is defined recursively in the following way. When $g=0, \vec{F}_{0}$ has two nodes, denoted by $a$ and $b$, and the weights of arcs $\vec{e}(a, b)$ and $\vec{e}(b, a)$ are defined to be $W_{a b}(0)$ $=W_{b a}(0)=1$. When $g \geq 1$, by construction, $F_{g}$ is obtained from $F_{g-1}$ by substituting each undirected edge $e(u, v)$ in $F_{g-1}$ with two undirected edges $e(u, w)$ and $e(w, v)$, and generating two additional nodes, $x$ and $y$, attaching to $u$ and $v$, respectively. The weights of resultant arcs in $\vec{F}_{g}$ are defined as: $W_{u w}(g)=W_{u v}(g-1), W_{v w}(g)=W_{v u}(g-$ $1), W_{w u}(g)=W_{w v}(g)=1, W_{x u}(g)=1, W_{y v}(g)=1, W_{u x}(g)=$ $\theta W_{u v}(g-1)$, and $W_{v y}(g)=\theta W_{v u}(g-1)$. Here $\theta$ is a tunable positive real number, that is, $\theta>0$. The weight parameter is of paramount importance since it characterizes the weight reciprocity of network $\vec{F}_{g}$. When $\theta=1, \vec{F}_{g}$ reduces to $F_{g}$, and the weights in two directions between any pair of adjacent nodes are completely reciprocated; when $\theta \neq 1$, the weights are non-reciprocated ${ }^{30-32}$ : the larger the deviation of $\theta$ from 1 , the smaller the level of weight reciprocity.

In fact, the introduced weight parameter $\theta$ acts as a similar role of energetic funnel in dendrimers controlling the MFPT to the center $^{39-41}$. It has been shown theoretically ${ }^{39,42}$ that the MFPT of Cayley trees as models of dendrimers can be influenced by both geometrical and energetic features. However, for complex systems with scale-free and fractal properties, it is still not well-understood how to superimpose an energetic funnel on their topological architecture, so that the MFPT depends on the interplay between structural and energetic properties. The main purpose of this work is to fill the gap, uncovering the collective impacts of topological and energetic aspects on dynamical processes, especially MFPT of random walks.

In undirected weighted networks ${ }^{43}$, node strength is a key quantity characterizing the property of a node. Here we extend the definition of strength of a node to the directed weighted network $\vec{F}_{g}$ by defining the out-strength and in-strength of node $i$ in $\vec{F}_{g}$ as $s_{i}^{+}(g)=\sum_{j=1}^{N_{g}} W_{i j}(g)$ and $s_{i}^{-}(g)=\sum_{j=1}^{N_{g}} W_{j i}(g)$, respectively. For $\vec{F}_{g}$, we can obtain the out-strength for an arbitrary node $i$ that entered the network at generation $g_{i}\left(g_{i} \geq 0\right)$. If $i$ was an external node when it entered the network, $s_{i}^{+}(g)=(\theta+1)^{g-g_{i}}$; otherwise, if $i$ was an internal node when it was born, $s_{i}^{+}(g)=2(\theta+1)^{g-g_{i}}$. Therefore, after each new iteration, the out-strength of a node increases by a factor of $\theta$. It is easy to obtain that the node out-strength in $\vec{F}_{g}$ obeys a distribution of power law form with the exponent being $1+\frac{2 \ln 2}{\ln (\theta+1)}$. Note that in some realistic networks, the node strength also display a broad distribution ${ }^{43}$.

Formulation of biased walks in the weighted directed network. After introducing the construction and properties of the weighted directed network $\vec{F}_{g}$, we now define and study biased discrete-time 
random walks performing $\vec{F}_{g}$. Let $r_{i j}(g)=W_{i j}(g) / s_{i}^{+}(g)$ denote the transition probability that a particle jumps from node $i$ to its neighboring node $j$ per time step. Note that $r_{i j}(g)$ constitutes an entry of transition matrix $R_{g}=\left(S_{g}\right)^{-1} W_{g}$, where $S_{g}$ is the diagonal out-strength matrix of $\vec{F}_{g}$, with the ith diagonal entry of $S_{g}$ being $s_{i}^{+}(g)$.

In this paper, we focus on a specific case of biased random walks, often called trapping problem, in $\vec{F}_{g}$ in the presence of a trap placed at the central hub node, i.e., the internal node generated at the first iteration. To facilitate the description of the following text, all the $N_{g}$ nodes in $\vec{F}_{g}$ are labeled sequentially as $1,2, \ldots, N_{g}-1, N_{g}$ as follows. For $\vec{F}_{1}$, the newly generated internal node is labeled 1 , the initial two nodes in $\vec{F}_{0}$ are labeled as 2 and 3, while the two new external nodes are labeled by 4 and 5 . For each new iteration $g_{i}>$ 1 , we label consecutively the new nodes born at this iteration from $N_{g_{i}-1}+1$ to $N_{g_{i}}$, while we keep the labels of those nodes created before iteration $g_{i}$ unchanged.

For the trapping problem, what we are concerned with are the trapping time and the average trapping time. Let $T_{i}^{(g)}$ represent the trapping time for a particle initially placed at node $i(i \neq 1)$ in $\vec{F}_{g}$ to arrive at the trap node for the first time, which is equal to the FPT from the $i$ to the trap. The average trapping time, $\langle T\rangle_{g}$, is actually the MFPT to the trap, defined as the mean of $T_{i}^{(g)}$ over all non-trap initial nodes in network $F_{g}$ :

$$
\langle T\rangle_{g}=\frac{1}{N_{g}-1} \sum_{i=2}^{N_{g}} T_{i}^{(g)} .
$$

Below we will show how to compute the two quantities $T_{i}^{(g)}$ and $\langle T\rangle_{g}$. For $T_{i}^{(g)}$, it obeys the relation

$$
T_{i}^{(g)}=1+\sum_{j=2}^{N_{g}} r_{i j} T_{j}^{(g)},
$$

which can be recast in matrix form as:

$$
T=e+\bar{R}_{g} T,
$$

where $T=\left(T_{2}^{(g)}, T_{3}^{(g)}, \ldots, T_{N_{g}}^{(g)}\right)^{\top}$ is an $\left(N_{g}-1\right)$-dimensional vector, $e=(1,1, \ldots, 1)^{\mathrm{T}}$ is the $\left(N_{g}-1\right)$-dimensional vector of all ones, and $\bar{R}_{g}$ is a matrix of order $N_{g}-1$, which a submatrix of $R_{g}$ and obtained from $R_{g}$ by deleting the first row and the first column corresponding to the trap. From equation (3) we have

$$
T=\left(I-\bar{R}_{g}\right)^{-1} e=K_{g} e,
$$

where $I$ is the $\left(N_{g}-1\right) \times\left(N_{g}-1\right)$ identity matrix, matrix $K_{g}=\left(I-\bar{R}_{g}\right)^{-1}$ is the fundamental matrix ${ }^{44}$ of the addressed trapping problem. Equation (4) implies

$$
T_{i}^{(g)}=\sum_{j=2}^{N_{g}} K_{g}(i, j),
$$

where $K_{g}(i, j)$ is the $i j$ th entry of matrix $K_{g}$, representing the expected number of visitations to node $j$ by a particle starting from node $i$ before being absorbed by the trap. Plugging equation (5) into equation (1) yields

$$
\langle T\rangle_{g}=\frac{1}{N_{g}-1} \sum_{i=2}^{N_{g}} \sum_{j=2}^{N_{g}} K_{g}(i, j) .
$$

Equation (6) indicates that the computation of MFPT $\langle T\rangle_{g}$ can be reduced to finding the sum of all entries of the corresponding fundamental matrix. A disadvantage of this method is that it demands a large computational effort when the network is very large. However, equation (6) provides exact results for $\langle T\rangle_{g}$ that can be applied to check the results for MFPT obtained using other techniques. Next we analytically determine the closed-form expression for MFPT $\langle T\rangle_{g}$ using an alternative approach, the results of which are consistent with those of equation (6). It should be noted that MFPT for other deterministic scale-free unweighed networks have been previously addressed ${ }^{45-47}$.

Exact solution to the MFPT $\langle\boldsymbol{T}\rangle_{g}$. The particular selection of trap location and the specific network structure allow to determine exactly the MFPT $\langle T\rangle_{g}$ for arbitrary $g$. In order to obtain a closeform expression for $\langle T\rangle_{g}$, we first establish the dependence of $T_{i}^{(g)}$ on iteration $g$. For a node $i$ in $\vec{F}_{g}$, at iteration $g+1$, its degree doubles, increasing from $k_{i}(g)$ to $2 k_{i}(g)$. All these $2 k_{i}(g)$ neighboring nodes are created at iteration $g+1$, among which one half are external nodes with a single degree, and the other half are internal nodes with degree 2 .

We now consider the trapping problem in $\vec{F}_{g+1}$. Let $A$ be the FPT for a particle starting from node $i$ to any of its $k_{i}(g)$ old neighbors, that is, those nodes adjacent to $i$ at iteration $g$; let $B$ (resp. $C$ ) be the FPT for a particle staring from any of the $k_{i}(g)$ internal (resp. external) neighbors of $i$ to one of its $k_{i}(g)$ old neighbors. Then the FPTs obey relations:

$$
\left\{\begin{array}{l}
A=\frac{\theta}{\theta+1}(1+C)+\frac{1}{\theta+1}(1+B), \\
B=\frac{1}{2}+\frac{1}{2}(1+A), \\
C=1+A .
\end{array}\right.
$$

Eliminating $B$ and $C$ in equation (7), we obtain $A=4(\theta+1)$. Therefore, when the network grows from iteration $g$ to iteration $g$ +1 , the FPT from any node $i\left(i \in \vec{F}_{g}\right)$ to another node $j\left(j \in \vec{F}_{g}\right)$ increases by a factor of $4(\theta+1)$. Hence, $T_{i}^{(g+1)}=4(\theta+1) T_{i}^{(g)}$ hold for any $g$, which is a useful for deriving the exact expression for MFPT.

Having obtained the scaling dominating the evolution for FPTs, we continue determining the MFPT $\langle T\rangle_{g}$. For this purpose, we introduce two intermediary quantities for any $n \leq g: T_{n \text {,tot }}^{(g)}=\sum_{i \in \Lambda_{n}} T_{i}^{(g)}$ and $\bar{T}_{n, \text { tot }}^{(g)}=\sum_{i \in \bar{\Lambda}_{n}} T_{i}^{(g)}$. Then,

$$
T_{g, \text { tot }}^{(g)}=T_{g-1, \text { tot }}^{(g)}+\bar{T}_{g, \text { tot }}^{(g)}=(4 \theta+4) T_{g-1, \text { tot }}^{(g-1)}+\bar{T}_{g, \text { tot }}^{(g)} .
$$

By definition, $\langle T\rangle_{g}=\frac{1}{N_{g}-1} T_{g \text {,tot }}^{(g)}$. To find $T_{g \text {,tot }}^{(g)}$, it is necessary to explicitly determine the quantity $\bar{T}_{g \text {,tot }}^{(g)}$. To this end, we define two additional quantities for $n \leq g$ : $\bar{T}_{n, \text { int }}^{(g)}=\sum_{i \in \bar{\Lambda}_{n, \text { int }}} T_{i}^{(g)}$ and $\bar{T}_{n \text {,ext }}^{(g)}=\sum_{i \in \bar{\Lambda}_{n, \text { ext }}} T_{i}^{(g)}$. Obviously, $\bar{T}_{g \text {,tot }}^{(g)}=\bar{T}_{g \text {,int }}^{(g)}+\bar{T}_{g, \text { ext }}^{(g)}$. Thus, in order to find $\bar{T}_{g \text {,tot }}^{(g)}$, one may alternatively evaluate $\bar{T}_{n \text {,int }}^{(g)}$ and $\bar{T}_{n \text {,ext }}^{(g)}$.

We first establish the relationship between $\bar{T}_{n \text {,int }}^{(g)}$ and $\bar{T}_{n \text {,ext }}^{(g)}$. By construction (see Fig. 1), at a given generation, each edge connecting two nodes $u$ and $v$ will give rise three new nodes $(w, x$, and $y)$ in the next generation. The two external nodes $x$ and $y$ are separately attached to $u$ and $v$, while the only internal node $w$ is linked simultaneously to $u$ and $v$. For any iteration $g$, the FPTs for the three new nodes satisfy: $T_{x}^{(g)}=1+T_{u}^{(g)}, \quad T_{y}^{(g)}=1+T_{v}^{(g)}$, and $T_{w}^{(g)}=\frac{1}{2}\left[1+T_{u}^{(g)}\right]+\frac{1}{2}\left[1+T_{v}^{(g)}\right]$. Therefore, $T_{x}^{(g)}+T_{y}^{(g)}=2 T_{w}^{(g)}$. Summing this relation over all old edges at the generation before growth, we find that for all $n \leq g, \bar{T}_{n \text {, ext }}^{(g)}=2 \bar{T}_{n \text {,int }}^{(g)}$ always holds. In 
this way, the issue of determining $\bar{T}_{g \text {,tot }}^{(g)}$ is reduced to finding $\bar{T}_{g \text {,ext }}^{(g)}$ that can be obtained as follows.

For an arbitrary external node $i_{\text {ext }}$ in $\vec{F}_{g}$, which is created at generation $g$ and attached to an old node $i$, we have $T_{i_{\text {ext }}^{(g)}}^{(g)}=1+T_{i}^{(g)}$, a relation valid for any node pair containing an old node and one of its new external adjacent nodes. By applying relation $T_{i_{\text {ext }}}^{(g)}=1+T_{i}^{(g)}$ to two sum (the first one is over a given old node and all its new adjacent external nodes, the other is summing the first one over all old nodes), we obtain

$$
\begin{aligned}
\bar{T}_{g, \text { ext }}^{(g)}= & \left|\bar{\Lambda}_{g, \text { ext }}\right|+\sum_{i \in \Lambda_{g-1}}\left(k_{i}(g-1) \times T_{i}^{(g)}\right) \\
= & \left|\bar{\Lambda}_{g, \text { ext }}\right|+\left(\bar{T}_{g-1, \text { ext }}^{(g)}+2 \bar{T}_{g-1, \text { int }}^{(g)}\right) \\
& +\left(2 \bar{T}_{g-2, \mathrm{ext}}^{(g)}+4 \bar{T}_{g-2, \text { int }}^{(g)}\right)+\cdots \\
& +\left(2^{g-2} \bar{T}_{1, \mathrm{ext}}^{(g)}+2^{g-1} \bar{T}_{1, \mathrm{int}}^{(g)}\right) \\
= & 2 \times 4^{g-1}+2 \bar{T}_{g-1, \mathrm{ext}}^{(g)}+4 \bar{T}_{g-2, \mathrm{ext}}^{(g)}+\cdots+2^{g-1} \bar{T}_{1, \mathrm{ext}}^{(g)}
\end{aligned}
$$

From equation (9), one can derive the recursive relation

$$
\bar{T}_{g+1, \text { ext }}^{(g+1)}=16(\theta+1) \bar{T}_{g, \text { ext }}^{(g)}-(4 \theta+2) 4^{g} .
$$

Considering the initial condition $\bar{T}_{2 \text {,ext }}^{(2)}=48 \theta^{2}+80 \theta+40$, equation (10) is solved to yield

$$
\bar{T}_{g, \text { ext }}^{(g)}=\frac{12 \theta^{2}+17 \theta+7}{(\theta+1)(4 \theta+3)} 2^{4_{g}-4}(\theta+1)^{g}+\frac{2 \theta+1}{4 \theta+3} 2^{2_{g}-1} .
$$

Because $\bar{T}_{g \text {,tot }}^{(g)}=\bar{T}_{g \text {,int }}^{(g)}+\bar{T}_{g \text {,ext }}^{(g)}$ and $\bar{T}_{g \text {,ext }}^{(g)}=2 \bar{T}_{g \text {,int }}^{(g)}$, we have

$$
\bar{T}_{g, \text { tot }}^{(g)}=\frac{36 \theta^{2}+51 \theta+21}{(\theta+1)(4 \theta+3)} 2^{4_{g}-5}(\theta+1)^{g}+\frac{6 \theta+3}{4 \theta+3} 2^{2_{g}-2} .
$$

Inserting equation (12) into equation (8) leads to

$$
\begin{aligned}
T_{g, \text { tot }}^{(g)}= & (4 \theta+4) T_{g-1, \text { tot }}^{(g-1)}+\frac{6 \theta+3}{4 \theta+3} 2^{2_{g}-2} \\
& +\frac{36 \theta^{2}+51 \theta+21}{(\theta+1)(4 \theta+3)} 2^{4_{g}-5}(\theta+1)^{g} .
\end{aligned}
$$

Using $T_{1, \text { tot }}^{(1)}=8 \theta+6$, equation (13) is solved to get

$$
\begin{aligned}
T_{g, \text { tot }}^{(g)}= & \frac{12 \theta^{3}+17 \theta^{2}+7 \theta}{\theta(\theta+1)(4 \theta+3)} 2^{4_{g}-3}(\theta+1)^{g} \\
& +\frac{16 \theta^{3}+28 \theta^{2}+20 \theta+6}{\theta(\theta+1)(4 \theta+3)} 2^{2_{g}-3}(\theta+1)^{g} \\
& -\frac{3(\theta+1)(2 \theta+1)}{\theta(\theta+1)(4 \theta+3)} 2^{2_{g}-2} .
\end{aligned}
$$

Then, the rigorous expression for the MFPT $\langle T\rangle_{g}$ of the weighted directed network $\vec{F}_{g}$ is

$$
\begin{aligned}
\langle T\rangle_{g}= & \frac{12 \theta^{3}+17 \theta^{2}+7 \theta}{8 \theta(\theta+1)(4 \theta+3)} 2^{2_{g}}(\theta+1)^{g} \\
& +\frac{16 \theta^{3}+28 \theta^{2}+20 \theta+6}{8 \theta(\theta+1)(4 \theta+3)}(\theta+1)^{g}-\frac{3(\theta+1)(2 \theta+1)}{4 \theta(\theta+1)(4 \theta+3)} .
\end{aligned}
$$

We have checked the analytical solution in equation (15) against extensive numerical results obtained from equation (6), see Fig. 3. For different $\theta$ and $g$, both the analytical and numerical results are in full agreement with each other, indicating that the explicit expression in equation (15) is correct. In addition, for the particular case $\theta=1$, the network $\vec{F}_{g}$ is reduced to $F_{g}$, and equation (15) recovers the result ${ }^{23}$ previously obtained for $F_{g}$. This also validates equation (15).

We proceed to express $\langle T\rangle_{g}$ in terms of the network size $N_{g}$, in order to uncover how $\langle T\rangle_{g}$ scales with $N_{g}$. From $N_{g}=4^{g}+1$, we have $g=\log _{4}\left(N_{g}-1\right)$. Then,

$$
\begin{aligned}
\langle T\rangle_{g}= & \frac{12 \theta^{3}+17 \theta^{2}+7 \theta}{8 \theta(\theta+1)(4 \theta+3)}\left(N_{g}-1\right)^{1+\log _{4}(\theta+1)} \\
& +\frac{16 \theta^{3}+28 \theta^{2}+20 \theta+6}{8 \theta(\theta+1)(4 \theta+3)}\left(N_{g}-1\right)^{\log _{4}(\theta+1)} \\
& -\frac{3(\theta+1)(2 \theta+1)}{4 \theta(\theta+1)(4 \theta+3)} .
\end{aligned}
$$

For a very large network (i.e., $N_{g} \rightarrow \infty$ ), the leading term of $\langle T\rangle_{g}$ can be represented as:

$$
\langle T\rangle_{g} \sim\left(N_{g}\right)^{1+\log _{4}(\theta+1)} .
$$

Equation (17) shows that for the directed weighted network $\vec{F}_{g}$, the MFPT $\langle T\rangle_{g}$ behaves as a power-law function of the network size $N_{g}$, with the exponent $\eta(\theta)=1+\log _{4}(\theta+1)$ increasing with the weight parameter $\theta$. Thus, the weight reciprocity has an essential effect on the efficiency of the trapping problem, measured by the MFPT.

Eigenvalues of the fundamental matrix. We now study the eigenvalues of the fundamental matrix $K_{n}$ of the trapping problem addressed above. We will determine all the eigenvalues of the fundamental matrix as well as their multiplicities. Moreover, we will show that the largest eigenvalue has the same leading scaling as that of $\langle T\rangle_{g}$. To attain this goal, we introduce matrix $P_{g}$ defined by $P_{g}=K_{g}^{-1}$. Let $\lambda_{i}(g)$ and $\sigma_{i}(g)$, where $i=1,2, \ldots, N_{n}-1$, denote the eigenvalues of $P_{g}$ and $K_{g}$, such that $\lambda_{1}(g) \leq \lambda_{2}(g) \leq \lambda_{3}(g) \ldots$ $\leq \lambda_{N_{g}-1}(g)$ and $\sigma_{1}(g) \geq \sigma_{2}(g) \geq \sigma_{3}(g) \geq \ldots \geq \sigma_{N_{n}-1}(g)$. Then, the one-to-one relation $\lambda_{i}(g)=1 / \sigma_{i}(g)$ holds. Thus, to compute the eigenvalues of matrix $K_{n}$, we can alternatively determine the eigenvalues for $P_{g}$. In the sequel, we will use the decimation method $^{48,49}$ to find all the eigenvalues of matrix $P_{g}$.

Full spectrum of fundamental matrix. The decimation procedure ${ }^{48,49}$ makes it possible to obtain the eigenvalues for related matrix of the current iteration from those of the previous iteration.

We now consider the eigenvalue problem for matrix $P_{g+1}$. Let $\alpha$ denote the set of nodes in network $\vec{F}_{g}$, and $\beta$ the set of nodes created at iteration $g+1$. Suppose that $\lambda_{i}(g+1)$ is an eigenvalue of $P_{g+1}$, and $u=\left(u_{\alpha}, u_{\beta}\right)^{\top}$ is an eigenvector associated with $\lambda_{i}(g+1)$, where $u_{\alpha}$ and $u_{\beta}$ correspond to nodes belonging to sets $\alpha$ and $\beta$, respectively. Then, eigenvalue equation for matrix $P_{g+1}$ can be represented in a block form:

$$
\left[\begin{array}{ll}
P_{\alpha, \alpha} & P_{\alpha, \beta} \\
P_{\beta, \alpha} & P_{\beta, \beta}
\end{array}\right]\left[\begin{array}{l}
u_{\alpha} \\
u_{\beta}
\end{array}\right]=\lambda_{i}(g+1)\left[\begin{array}{l}
u_{\alpha} \\
u_{\beta}
\end{array}\right],
$$

where $P_{\alpha, \alpha}$ and $P_{\beta, \beta}$ are the identity matrix.

Equation (18) can be expressed as two equations:

$$
\begin{aligned}
& P_{\alpha, \alpha} u_{\alpha}+P_{\alpha, \beta} u_{\beta}=\lambda_{i}(g+1) u_{\alpha}, \\
& P_{\beta, \alpha} u_{\alpha}+P_{\beta, \beta} u_{\beta}=\lambda_{i}(g+1) u_{\beta} .
\end{aligned}
$$

Equation (20) implies

$$
u_{\beta}=\frac{1}{\lambda_{i}(g+1)-1} P_{\beta, \alpha} u_{\alpha}
$$


provided that $\lambda_{i}(g+1) \neq 1$. Inserting equation (21) into equation (19) yields

$$
P_{\alpha, \beta} P_{\beta, \alpha} u_{\alpha}=\left[\lambda_{i}(g+1)-1\right]^{2} u_{\alpha}
$$

In this way, we reduce the problem of determining the eigenvalue $\lambda_{i}(g$ +1 ) for matrix $P_{g+1}$ of order $4^{g+1}$ to finding the eigenvalue problem of matrix $P_{\alpha, \beta} P_{\beta, \alpha}$ with a smaller order $4^{g}$.

We can prove (see Methods) that

$$
P_{\alpha, \beta} P_{\beta, \alpha}=I_{g}-\frac{1}{2 \theta+2} P_{g},
$$

where $I_{g}$ is the identity matrix of order $4^{g}$, identical to that of $P_{g}$. Equation (23) relates the product matrix $P_{\alpha, \beta} P_{\beta, \alpha}$ to matrix $P_{g}$. Therefore, the eigenvalues of matrix $P_{g+1}$ can be expressed in terms of those of matrix $P_{g}$.

We next show how to obtain the eigenvalues of $P_{g+1}$ through the eigenvalues of $P_{g}$. According to equations (22) and (23), we can derive

$$
P_{g} u_{\alpha}=-(2 \theta+2)\left[\lambda_{i}^{2}(g+1)-2 \lambda_{i}(g+1)\right] u_{\alpha} .
$$

Hence, if $\lambda_{i}(g)$ is an eigenvalue of $P_{g}$ associated with eigenvector $u_{a}$, equation (24) indicates

$$
\lambda_{i}(g)=-(2 \theta+2)\left[\lambda_{i}(g+1)^{2}-2 \lambda_{i}(g+1)\right] .
$$

Solving the above quadratic equation in the variable $\lambda_{i}(g+1)$ given by equation (25), one obtains the two roots:

$$
\lambda_{i, 1}(g+1)=1-\sqrt{1-\frac{\lambda_{i}(g)}{2 \theta+2}},
$$

and

$$
\lambda_{i, 2}(g+1)=1+\sqrt{1-\frac{\lambda_{i}(g)}{2 \theta+2}} .
$$

Equations (26) and (27) relate $\lambda_{i}(g+1)$ to $\lambda_{i}(g)$, with each eigenvalue $\lambda_{i}(g)$ of $P_{g}$ giving rise two different eigenvalues of $P_{g+1}$. As a matter of fact, all eigenvalues of the $P_{g+1}$ can be obtained by these two recursive relations. In Methods, we determine the multiplicity of each eigenvalue and show that all the eigenvalues can be found by equations (26) and (27).

Since there is a one-to-one relation between the eigenvalues of $P_{g}$ and the fundamental matrix $K_{g}$, we thus have also found all the eigenvalues of $K_{g}$.

The largest eigenvalue of fundamental matrix and MFPT. In the above, we have determined all eigenvalues for the inverse $P_{g}$ of the fundamental matrix $K_{g}$ and thus all eigenvalues of $K_{g}$. Here we continue to estimate the greatest eigenvalue $\sigma_{\max }(g)$ of the fundamental matrix $K_{g}$, which actually equals the reciprocal of the smallest eigenvalue for matrix $P_{g}$, denoted by $\lambda_{\min }(g)$. Below we will show that in a large network the leading behavior of the MFPT $\langle T\rangle_{g}$ for trapping in $\vec{F}_{g}$ and the reciprocal of $\lambda_{\text {min }}(g)$ is identical, that is, $\langle T\rangle_{g} \sim 1 / \lambda_{\min }(g)=\sigma_{\max }(g)$.

We begin by providing some useful properties of eigenvalues for matrix $P_{g}$. Assume that $\Delta_{g}$ is the set of the $4^{g}$ eigenvalues of matrix $P_{g}$, namely, $\Delta_{g}=\left\{\lambda_{1}(g), \lambda_{2}(g), \lambda_{3}(g), \cdots, \lambda_{4}(g)\right\}$. According to the above analysis, $\Delta_{g}$ can be categorized into two subsets $\Delta_{g}^{(1)}$ and $\Delta_{g}^{(2)}$ satisfying $\Delta_{g}=\Delta_{g}^{(1)} \cup \Delta_{g}^{(2)}$, where $\Delta_{g}^{(1)}$ consists of all eigenvalues 1 , while $\Delta_{g}^{(2)}$ contains the rest eigenvalues. Thus,

$$
\Delta_{g}^{(1)}=\underbrace{\{1,1,1, \ldots, 1,1\}}_{2 \times 4^{g-1}} .
$$

These $2 \times 4^{g-1}$ eigenvalues are labeled sequentially by $\lambda_{4^{g-1}+1}(g), \lambda_{4^{g-1}+2}(g), \cdots, \lambda_{3 \times 4^{g-1}}(g)$, since they provide a natural increasing order of all eigenvalues for $P_{g}$, as will be shown.

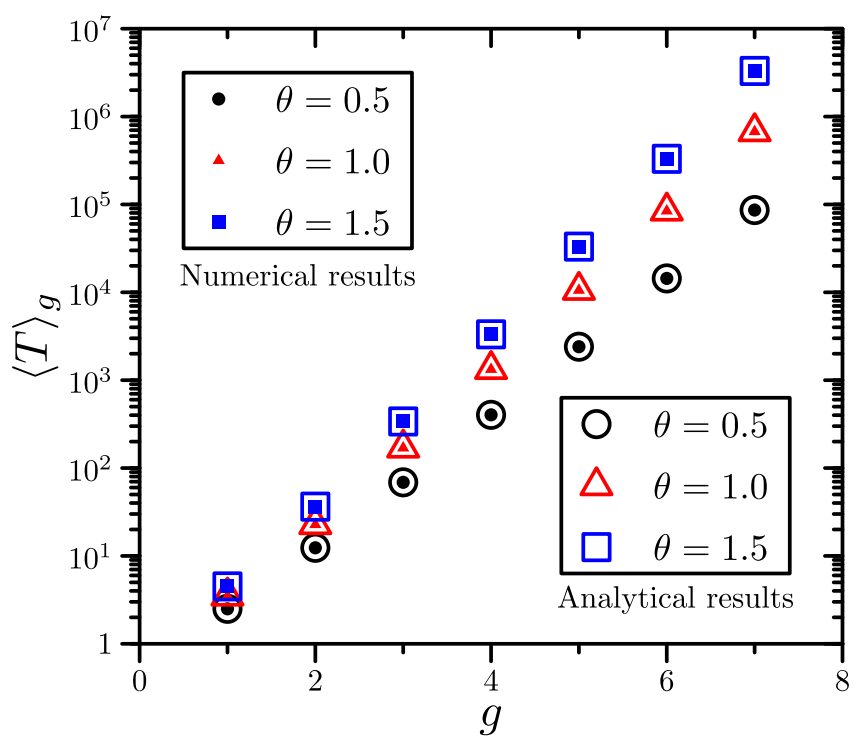

Figure $3 \mid$ MFPT $\langle T\rangle_{g}$ as a function of $g$ for different networks with various $\theta$. The filled symbols are the data obtained by direct calculation from equation (6); while the empty symbols are those exact analytical values given by equation (15).

The remaining $2 \times 4^{g-1}$ eigenvalues in set $\Delta_{g}^{(2)}$ are all determined by equations (26) and (27). Let $\lambda_{1}(g-1), \lambda_{2}(g-1), \lambda_{3}(g-1)$, $\cdots, \lambda_{4^{g-1}}(g-1)$ be the $4^{g-1}$ eigenvalues of matrix $P_{g-1}$, arranged in an increasing order $\lambda_{1}(g-1) \leq \lambda_{2}(g-1) \leq \lambda_{3}(g-1) \leq \ldots$ $\leq \lambda_{4^{g-1}}(g-1)$. Then, for each eigenvalue $\lambda_{i}(g-1)$ of $P_{g-1}$, equations (26) and (27) produce two eigenvalues of $P_{g}$, which are labeled as $\lambda_{i}(g)$ and $\lambda_{4 g-i+1}(g)$ :

$$
\lambda_{i}(g)=1-\sqrt{1-\frac{\lambda_{i}(g-1)}{2 \theta+2}}
$$

and

$$
\lambda_{4 g-i+1}(g)=1+\sqrt{1-\frac{\lambda_{i}(g-1)}{2 \theta+2}} .
$$

Plugging each eigenvalue of $P_{g-1}$ into equations (26) and (27) generates all eigenvalues in $\Delta_{g}^{(2)}$.

It is easy to see that $\lambda_{i}(g)$ given by equation (29) monotonously increases with $\lambda_{i}(g-1)$ and belongs to interval $(0,1)$, while $\lambda_{4^{g-i+1}}(g)$ provided by equation (30) monotonously decreases with $\lambda_{i}(g-1)$ and lies in interval $(1,2)$. Thus, $\lambda_{1}(g), \lambda_{2}(g), \lambda_{3}(g), \cdots, \lambda_{4^{g}}(g)$ provide an increasing order of all eigenvalues for matrix $P_{g}$.

We hasten to estimate $\lambda_{\min }(g)$ of matrix $P_{g}$. From the above arguments, the smallest eigenvalue $\lambda_{\min }(g)$ must be the one generated from $\lambda_{\min }(g-1)$ through equation (29):

$$
\lambda_{\min }(g)=1-\sqrt{1-\frac{\lambda_{\min }(g-1)}{2 \theta+2}} .
$$

Using Taylor's formula, we have

$$
\lambda_{\text {min }}(g) \approx 1-\left[1-\frac{\lambda_{\min }(g-1)}{4 \theta+4}\right]=\frac{\lambda_{\min }(g-1)}{4 \theta+4} .
$$

Considering $\lambda_{\min }(1)=1-\sqrt{1-\frac{1}{\theta+1}}$, equation (32) is solved to yield

$$
\lambda_{\min }(g) \approx\left(1-\sqrt{1-\frac{1}{\theta+1}}\right)(4 \theta+4)^{1-g} .
$$


Thus,

$$
\frac{1}{\lambda_{\min }(g)} \approx \frac{1}{4(\theta+1)^{2}}\left(1+\sqrt{1-\frac{1}{\theta+1}}\right) 4^{g}(\theta+1)^{g},
$$

which, together with equation (15), means that $\frac{1}{\lambda_{\min }(g)}$ and $\langle T\rangle_{g}$ have the same dominating term and thus identical leading scaling.

\section{Discussion}

Real-life weighted networks exhibit a rich and diverse reciprocity structure. In this paper, we have proposed a scale-free weighted directed network with asymmetric edge weights, which are controlled by a parameter characterizing the network reciprocity. We then studied random walks performed on the network with a trap fixed at the central hub node. Applying two different approaches, we have evaluated the MFPT to the trap. Moreover, based on the selfsimilar architecture of the network, we have found all the eigenvalues and their multiplicities of the fundamental matrix describing the random-walk process, the largest one of which has the same leading scaling as that of the MFPT. The obtained results indicate that the MFPT scales as a power-law function of the the system size, with the power exponent increasing with the weight parameter, revealing that the reciprocity has a significant impact on dynamical processes running on weighted networks. Finally, it should be mentioned that although we have only studied a particular network, our methods can also be applied to other self-similar networks, obtaining analogous results. Thus, our work deepens the understanding of random-walk dynamics in complex systems and opens a novel avenue to control random walks in a weighted network by changing its reciprocity.

\section{Methods}

Proof of equation (23). In order to prove equation (23), we rewrite $P_{\alpha, \beta}$ and $P_{\beta, \alpha}$ in the block form as

$$
P_{\alpha, \beta}=\left(U_{1}, U_{2}, \cdots, U_{E_{g}}\right)
$$

and

$$
P_{\beta, \alpha}=\left(\begin{array}{c}
V_{1} \\
V_{2} \\
\vdots \\
V_{E_{g}}
\end{array}\right),
$$

respectively. In equations (35) and (36), $E_{g}=4^{g}$ is the number of edges in $F_{g} ; U_{i}(1 \leq i$ $\left.\leq E_{g}\right)$ is a $4^{g} \times 3$ matrix describing the transition probability from the $4^{g}$ non-trap nodes of $F_{g}$ to the three nodes newly generated by the $i$ th edge of $F_{g}$; similarly, $V_{i}(1 \leq i$ $\leq E_{g}$ ) is a $3 \times 4^{g}$ matrix indicating the transition probability from the three new nodes created by the $i$ th edge to those $4^{g}$ old non-trap nodes belonging to $F_{g}$. Then,

$$
\begin{aligned}
& P_{\alpha, \beta} P_{\beta, \alpha}=\sum_{i=1}^{E_{g}} U_{i} V_{i} \\
= & \sum_{i=1}^{E_{g}}\left(\frac{a_{i}}{\theta+1} \varepsilon_{l_{i}}+\frac{b_{i}}{\theta+1} \varepsilon_{r_{i}}, \frac{\theta}{\theta+1} a_{i} \varepsilon_{l_{i}}, \frac{\theta}{\theta+1} b_{i} \varepsilon_{r_{i}}\right) \times \\
& \left(\begin{array}{c}
-\frac{\varepsilon_{l_{i}}^{\top}+\varepsilon_{r_{i}}^{\top}}{2} \\
-\varepsilon_{l_{i}}^{\top} \\
-\varepsilon_{r_{i}}^{\top}
\end{array}\right) \\
= & -\frac{1}{2 \theta+2} \times \\
& \sum_{i=1}^{E_{g}}\left[(2 \theta+1)\left(a_{i} \varepsilon_{l_{i}} \varepsilon_{l_{i}}^{\top}+b_{i} \varepsilon_{r_{i}} \varepsilon_{r_{i}}^{\top}\right)+a_{i} \varepsilon_{l_{i}} \varepsilon_{r_{i}}^{\top}+b_{i} \varepsilon_{r_{i}} \varepsilon_{l_{i}}^{\top}\right] \\
= & I_{g}-\frac{1}{2 \theta+2} P_{g},
\end{aligned}
$$

which completes the proof of equation (23). Note that in equation (37), $l_{i}$ and $r_{i}$ are the two endpoints of the $i$ th edge of $F_{g}$; $\varepsilon_{i}$ is a vector having only one nonzero element 1 at $i$ th entry with other entries being zeros; $a_{i}$ and $b_{i}$ are two entries of $P_{g}$ corresponding to edges $\left(l_{i}, r_{i}\right)$ and $\left(r_{i}, l_{i}\right)$, respectively.

Alternative proof of equation (23). Equation (23) can also be proved using another technique. Assume that $R_{g}=P_{\alpha, \beta} P_{\beta, \alpha}$ and $Q_{g}=I_{g}-\frac{1}{2 \theta+2} P_{g}$. In order to prove $P_{\alpha, \beta} P_{\beta, \alpha}=I_{g}-\frac{1}{2 \theta+2} P_{g}$, it suffices to show that the entries of $R_{g}$ are equal to their counterparts of $Q_{g}$. For matrix $Q_{g}$, it is easy to see that its entries are: $Q_{g}(i, i)=\frac{2 \theta+1}{2 \theta+2}$ for $i=j$ and $Q_{g}(i, j)=-\frac{1}{2 \theta+2} P_{g}(i, j)$ otherwise. If $P_{g+1}(i, j)$ denotes the $(i, j)$ entry of matrix $P_{g+1}$, the entries of $R_{g}(i, j)$ of matrix $R_{g}$ can be evaluated by distinguishing two cases: $i=j$ and $i \neq j$.

For the case of $i=j$, the diagonal element of $R_{g}$ is

$$
\begin{aligned}
& R_{g}(i, i)= \\
= & \sum_{z \in \beta} P_{g+1}(i, z) P_{g+1}(z, i)=\sum_{z \in \beta} \frac{W_{i z}(g+1)}{s_{i}^{+}(g+1)} \frac{W_{z i}(g+1)}{s_{z}^{+}(g+1)} \\
= & \frac{1}{2} \sum_{\substack{z \in \beta, i \sim z \\
k_{z}(g+1)=2}} \frac{W_{i z}(g+1)}{s_{i}^{+}(g+1)}+\sum_{\substack{z \in \beta, i \sim z \\
k_{z}(g+1)=1}} \frac{W_{i z}(g+1)}{s_{i}^{+}(g+1)} \\
= & \frac{1}{2} \frac{s_{i}^{+}(g)}{s_{i}^{+}(g+1)}+\frac{\theta s_{i}^{+}(g)}{s_{i}^{+}(g+1)} \\
= & \frac{2 \theta+1}{2 \theta+2}=Q_{g}(i, i),
\end{aligned}
$$

where the relation $s_{i}^{+}(g+1)=(\theta+1) s_{i}^{+}(g)$ is used. In equation (38), $i \sim z$ indicates that two nodes $i$ and $z$ are adjacent in network $F_{g+1}$.

For the other case of $i \neq j$, the non-diagonal element of $R_{g}$ is

$$
\begin{aligned}
R_{g}(i, j) & =\sum_{z \in \beta} P_{g+1}(i, z) P_{g+1}(z, j) \\
& =\sum_{\substack{A_{g+1}(i, z)=1 \\
A_{g+1}(z, j)=1}} \frac{W_{i z}(g+1)}{s_{i}^{+}(g+1)} \frac{W_{z j}(g+1)}{s_{z}^{+}(g+1)} \\
& =\frac{1}{2} \frac{W_{i j}(g)}{s_{i}^{+}(g+1)}=-\frac{1}{2 \theta+2} P_{g}(i, j) \\
& =Q_{g}(i, j),
\end{aligned}
$$

which, together with (38) proves equation (23).

Multiplicities of eigenvalues. By numerically computing the eigenvalues for the first several iterations, we can observe some important phenomena and properties about the structure of the eigenvalues. When $g=1$, the eigenvalues of $P_{1}$ are $1-\sqrt{1-\frac{1}{\theta+1}}$ and $1+\sqrt{1-\frac{1}{\theta+1}}$, both of which have a multiplicity of 2 . When $g=2, P_{2}$ have 16 eigenvalues: eigenvalue 1 with degeneracy 8 and 4 two-fold other eigenvalues generated by $1-\sqrt{1-\frac{1}{\theta+1}}$ and $1+\sqrt{1-\frac{1}{\theta+1}}$. When $g \geq 3$, all the eigenvalues $P_{g}$ can be put into two classes. The first class includes eigenvalue 1 and those generated by 1 , which display the following feature that each eigenvalue appearing at a given iteration $g_{i}$ will continue to appear at all subsequent generations greater than $g_{i}$. The second class contains those eigenvalues generated by the two eigenvalues

$1-\sqrt{1-\frac{1}{\theta+1}}$ and $1+\sqrt{1-\frac{1}{\theta+1}}$ of $P_{1}$. Each eigenvalue in this class is two-fold, and each eigenvalue of a given iteration $g_{i}$ does not appear at any of subsequent iterations larger than $g_{i}$. For the two eigenvalue classes, each eigenvalue (other than 1) of current generation keeps the multiplicity of its father of the previous generation.

Using the above-observed properties of the eigenvalue structure, we can determine the multiplicities of all eigenvalues. Let $M_{g}(\lambda)$ denote the multiplicity of eigenvalue $\lambda$ of matrix $P_{g}$. We first determine the number of eigenvalue 1 for $P_{g}$. To this end, let $r(X)$ denote the rank of matrix $X$. Then

$$
M_{g}(\lambda=1)=4^{g}-r\left(P_{g}-1 \times I_{g}\right) .
$$

For $g=1, M_{1}(1)=0$; for $g=2, M_{2}(1)=8$. For $g \geq 2$, it is obvious that $r\left(P_{g+1}-I_{g+1}\right)$ $=r\left(P_{\alpha, \beta}\right)+r\left(P_{\beta, \alpha}\right)$, where $r\left(P_{\alpha, \beta}\right)$ and $r\left(P_{\beta, \alpha}\right)$ can be determined in the following way. We first show that $P_{\beta, \alpha}$ is a full column rank matrix. Let

$$
\phi=\left(\phi_{1}, \phi_{2}, \cdots, \phi_{3 \times 48}\right)^{\top}=\sum_{\substack{i \in \alpha \\ i \neq 1}} k_{i} M_{i},
$$

where $M_{i}$ is the column vector of $P_{\beta, \alpha}$ representing the $i$ th column of $P_{\beta, \alpha}$. Let $M_{i}=\left(M_{1, i}, M_{2, i}, \cdots, M_{3 \times 48, i}\right)^{\top}$. Suppose that $\phi=0$. Then, we can prove that for an arbitrary $k_{i}, k_{i}=0$ always holds. By construction, for any old node $i \in \alpha$, there exists a 
new leaf node $l \in \beta$ attached to $i$. Then, for $\phi_{l}=k_{1} M_{1, l}+k_{2} M_{2, l}, \cdots,+k_{3 \times 48} M_{3 \times 48, l}$, only $M_{i, l} \neq 0$ but all $M_{x, l}=0$ for $x \neq i$. From $\phi_{l}=0$, we have $k_{i}=0$. Therefore, $r\left(P_{\beta, \alpha}\right)$ $=4^{g}$. Analogously, we can verify that $P_{\alpha, \beta}$ is a full row rank matrix and $r\left(P_{\alpha, \beta}\right)=4^{g}$. Combining the above results, the multiplicity of eigenvalue 1 of $P_{g}$ is

$$
M_{g}(\lambda=1)= \begin{cases}0, & g=1, \\ 2 \times 4^{g-1}, & g \geq 2 .\end{cases}
$$

We proceed to compute the multiplicities of other eigenvalues generated by 1 that are in the first eigenvalue class. Since every eigenvalue at a given iteration keeps the multiplicity of its father at the preceding iteration, for matrix $P_{g}$, the multiplicity of each first-generation descendant of eigenvalue 1 is $2 \times 4^{g-2}$, the multiplicity of each second-generation descendant of eigenvalue 1 is $2 \times 4^{g-3}$, and the multiplicity of each $(g-2)$ nd generation descendant of eigenvalue 1 is $2 \times 4$. Moreover, we can derive that that the number of the $i$ th $(0 \leq i \leq g-2)$ generation distinct descendants of eigenvalue is $2^{i}$, where 0 th generation descendants refer to the $2 \times 4^{g^{-1}}$ eigenvalues 1 themselves. Finally, it is easy to verify that the number of all the eigenvalues in the second eigenvalue class is $4 \times 2^{g-1}$. Hence, the total number of eigenvalues of matrix $P_{g}$ is

$$
\sum_{i=0}^{g-2}\left[\left(2 \times 4^{g-1-i}\right) \times 2^{i}\right]+4 \times 2^{g-1}=4^{g},
$$

indicating that all the eigenvalues of $P_{g}$ are successfully found.

1. Newman, M. E. J. The structure and function of complex networks. SIAM Rev. 45 167-256 (2003).

2. Kleinberg, J. M. Navigation in a small world. Nature 406, 845-845 (2000).

3. Guimerà, R., Diaz-Guilera, A., Vega-Redondo, F., Cabrales, A. \& Arenas, A Optimal network topologies for local search with congestion. Phys. Rev. Lett. 89, 248701 (2002)

4. Bénichou, O., Loverdo, C., Moreau, M. \& Voituriez, R. Intermittent search strategies. Rev. Mod. Phys. 83, 81-129 (2011).

5. Olfati-Saber, R., Fax, J. A. \& Murray, R. M. Consensus and cooperation in networked multiagent systems. Proceedings of the IEEE 95, 215-233 (2007).

6. Bartumeus, F., da Luz, M. G. E., Viswanathan, G. \& Catalan, J. Animal search strategies: a quantitative random-walk analysis. Ecology 86, 3078-3087 (2005).

7. Brockmann, D., Hufnagel, L. \& Geisel, T. The scaling laws of human travel. Nature 439, 462-465 (2006).

8. Weiss, G. H. Aspects and Applications of the Random Walk (North-Holland, Amsterdam, 2005)

9. Grady, L. Random walks for image segmentation. IEEE Trans. Pattern Analysis and Machine Intelligence 28, 1768-1783 (2006)

10. Pons, P. \& Latapy, M. Computing communities in large networks using random walks. J. Graph Algorithms Appl. 10, 191-218 (2006).

11. Rosvall, M., Esquivel, A. V., Lancichinetti, A., West, J. D. \& Lambiotte, R. Memory in network flows and its effects on spreading dynamics and community detection. Nat. Commun. 5, 4630 (2014).

12. Fouss, F., Pirotte, A., Renders, J.-M. \& Saerens, M. Random-walk computation of similarities between nodes of a graph with application to collaborative recommendation. IEEE Trans. Knowl. Data Eng. 19, 355-369 (2007).

13. Chennubhotla, C. \& Bahar, I. Signal propagation in proteins and relation to equilibrium fluctuations. PLoS Comput. Biol. 3, e172 (2007).

14. Redner, S. A guide to first-passage processes (Cambridge University Press, 2001).

15. Bénichou, O. \& Voituriez, R. From first-passage times of random walks in confinement to geometry-controlled kinetic. Phys. Rep. 539, 225-284 (2014).

16. Lin, Y. \& Zhang, Z. Mean first-passage time for maximal-entropy random walks in complex networks. Sci. Rep. 4, 5365 (2014).

17. Noh, J. D. \& Rieger, H. Random walks on complex networks. Phys. Rev. Lett. 92, $118701(2004)$

18. Condamin, S., Bénichou, O. \& Moreau, M. First-passage times for random walks in bounded domains. Phys. Rev. Lett. 95, 260601 (2005).

19. Condamin, S., Bénichou, O. \& Klafter, J. First-passage time distributions for subdiffusion in confined geometry. Phys. Rev. Lett. 98, 250602 (2007).

20. Condamin, S., Bénichou, O., Tejedor, V., Voituriez, R. \& Klafter, J. First-passage times in complex scale-invariant media. Nature 450, 77-80 (2007).

21. Zhang, Z. Z., Qi, Y., Zhou, S. G., Xie, W. L. \& Guan, J. H. Exact solution for mean first-passage time on a pseudofractal scale-free web. Phys. Rev. E 79, 021127 (2009).

22. Lin, Y. \& Zhang, Z. Z. Random walks in weighted networks with a perfect trap: An application of Laplacian spectra. Phys. Rev. E 87, 062140 (2013).

23. Zhang, Z., Xie, W., Zhou, S., Gao, S. \& Guan, J. Anomalous behavior of trapping on a fractal scale-free network. EPL 88, 10001 (2009).

24. Zhang, Z. et al. Trapping in scale-free networks with hierarchical organization of modularity. Phys. Rev. E 80, 051120 (2009).

25. Garlaschelli, D. \& Loffredo, M. I. Patterns of link reciprocity in directed networks. Phys. Rev. Lett. 93, 268701 (2004)
26. Albert, R., Jeong, H. \& Barabási, A.-L. Internet: Diameter of the World-Wide Web. Nature 401, 130-131 (1999).

27. Ebel, H., Mielsch, L.-I. \& Bornholdt, S. Scale-free topology of e-mail networks. Phys. Rev. E 66, 035103 (2002).

28. Newman, M. E., Forrest, S. \& Balthrop, J. Email networks and the spread of computer viruses. Phys. Rev. E 66, 035101 (2002).

29. Serrano, M. Á. \& Boguñá, M. Topology of the world trade web. Phys. Rev. E 68 , 015101 (2003).

30. Akoglu, L., Vaz de Melo, P. O. S. \& Faloutsos, C. Quantifying reciprocity in large weighted communication networks. Lect. Notes Comput. Sci. 7302, 85-96 (2012).

31. Wang, C. et al. A dyadic reciprocity index for repeated interaction networks. Netw. Sci. 1, 31-48 (2013).

32. Squartini, T., Picciolo, F., Ruzzenenti, F. \& Garlaschelli, D. Reciprocity of weighted networks. Sci. Rep. 3, 2729 (2013)

33. Zhu, Y. X. et al. Influence of reciprocal links in social networks. PLoS ONE 9 , e103007 (2014).

34. Boguñá, M. \& Serrano, M. Á. Generalized percolation in random directed networks. Phy. Rev. E 72, 016106 (2005).

35. Song, C., Havlin, S. \& Makse, H. A. Origins of fractality in the growth of complex networks. Nat. Phys. 2, 275-281 (2006).

36. Rozenfeld, H. D., Havlin, S. \& ben Avraham, D. Fractal and transfractal recursive scale-free nets. New J. Phys. 9, 175 (2007).

37. Barabási, A.-L. \& Albert, R. Emergence of scaling in random networks. Science 286, 509-512 (1999).

38. Song, C., Havlin, S. \& Makse, H. A. Self-similarity of complex networks. Nature 433, 392-395 (2005)

39. Bar-Haim, A., Klafter, J. \& Kopelman, R. Dendrimers as controlled artificial energy antennae. J. Am. Chem. Soc. 119, 6197-6198 (1997).

40. Bar-Haim, A. \& Klafter, J. Geometric versus energetic competition in light harvesting by dendrimers. J. Phys. Chem. B 102, 1662-1664 (1998).

41. Bar-Haim, A. \& Klafter, J. On mean residence and first passage times in finite onedimensional systems. J. Chem. Phys. 109, 5187-5193 (1998).

42. Kopelman, R. et al. Spectroscopic evidence for excitonic localization in fractal antenna supermolecules. Phys. Rev. Lett. 78, 1239-1242 (1997).

43. Barrat, A., Barthelemy, M., Pastor-Satorras, R. \& Vespignani, A. The architecture of complex weighted networks. Proc. Natl Acad. Sci. USA 101, 3747-3752 (2004)

44. Kemeny, J. G. \& Snell, J. L. Finite Markov chains (van Nostrand Princeton, NJ 1960).

45. Agliari, E. \& Burioni, R. Random walks on deterministic scale-free networks: Exact results. Phys. Rev. E 80, 031125 (2009).

46. Tejedor, V., Benichou, O. \& Voituriez, R. Close or connected: Distance and connectivity effects on transport in networks. Phys. Rev. E 83, 066102 (2011)

47. Meyer, B., Agliari, E., Benichou, O. \& Voituriez, R. Exact calculations of firstpassage quantities on recursive networks. Phys. Rev. E 85, 026113 (2012).

48. Domany, E., Alexander, S., Bensimon, D. \& Kadanoff, L. P. Solutions to the schrödinger equation on some fractal lattices. Phys. Rev. B 28, 3110 (1983).

49. Blumen, A., Von Ferber, C., Jurjiu, A. \& Koslowski, T. Generalized Vicsek fractals Regular hyperbranched polymers. Macromolecules 37, 638-650 (2004).

\section{Acknowledgments}

The authors thank Bin Wu for his assistance in preparing this manuscript. This work was supported by the National Natural Science Foundation of China under Grant No. 11275049. H. L. was also supported by Fudan's Undergraduate Research Opportunities Program.

\section{Author contributions}

Z.Z.Z. designed the research; H.L. and Y.B.S. performed the research; Z.Z.Z. and H.L. wrote the manuscript.

\section{Additional information}

Competing financial interests: The authors declare no competing financial interests.

How to cite this article: Zhang, Z., Li, H. \& Sheng, Y. Effects of reciprocity on random walks in weighted networks. Sci. Rep. 4, 7460; DOI:10.1038/srep07460 (2014).

This work is licensed under a Creative Commons Attribution-NonCommercialNoDerivs 4.0 International License. The images or other third party material in this article are included in the article's Creative Commons license, unless indicated otherwise in the credit line; if the material is not included under the Creative Commons license, users will need to obtain permission from the license holder in order to reproduce the material. To view a copy of this license, visit http:// creativecommons.org/licenses/by-nc-nd/4.0/ 